Case Report

\title{
Surgical Treatment for Severe Scoliosis in Patients with Reduced Cardiorespiratory Function after Surgery for Congenital Heart Disease: A Report of Two Cases
}

\author{
Hayato Kinoshita $\left(\mathbb{D},{ }^{1}\right.$ Naohisa Miyakoshi $\left(\mathbb{D},{ }^{2}\right.$ Michio Hongo $\mathbb{D}^{2},{ }^{2}$ Akiko Misawa, ${ }^{3}$ \\ Daisuke Kudo $\mathbb{D}^{2}{ }^{2}$ and Yoichi Shimada ${ }^{2}$ \\ ${ }^{1}$ Department of Orthopedic Surgery, Akita Kousei Medical Center, 1-1-1 Iijima-Nishibukuro, Akita 011-0948, Japan \\ ${ }^{2}$ Department of Orthopedic Surgery, Akita University Graduate School of Medicine, 1-1-1 Hondo, Akita 010-8543, Japan \\ ${ }^{3}$ Akita Prefectural Center on Development and Disability, 3-128 Kamikitate Hyakuzaki Suwanosawa, Akita 010-1407, Japan \\ Correspondence should be addressed to Hayato Kinoshita; hayato@med.akita-u.ac.jp
}

Received 29 May 2018; Revised 13 August 2018; Accepted 6 September 2018; Published 25 September 2018

Academic Editor: Ali F. Ozer

Copyright (c) 2018 Hayato Kinoshita et al. This is an open access article distributed under the Creative Commons Attribution License, which permits unrestricted use, distribution, and reproduction in any medium, provided the original work is properly cited.

Purpose. Congenital heart disease (CHD) is associated with an increased risk of scoliosis. The prognosis of scoliosis patients with $\mathrm{CHD}$ has improved because of advances in cardiac care. As a result, the frequency of surgery for scoliosis in this population has increased, although the risk of perioperative complications remains high. We treated two patients with CHD who underwent surgery for severe scoliosis. To avoid perioperative complications, we evaluated the preoperative cardiac status and anesthetic risks before posterior correction and fixation in both patients. Methods. An expert anesthesiologist evaluated the anesthetic risk in each case, and an adequate reservoir of autologous blood was collected preoperatively. The patient in case 1 was at risk of significant blood loss and required extremely careful operative technique. The patient in case 2 had low cardiac output preoperatively. We therefore performed a thorough preoperative cardiac evaluation. Both patients were admitted to the intensive care unit postoperatively. Results. Neither patient suffered serious complications, and both achieved favorable outcomes. Conclusions. Appropriate surgical technique and teamwork among experts are the keys to success in patients with severe scoliosis and CHD.

\section{Introduction}

Patients with congenital heart disease (CHD) are at increased risk of developing scoliosis. Van Biezen et al. reported that all patients who underwent left thoracotomy developed secondary scoliosis [1]. The exact mechanisms of scoliosis development, however, are unclear. In contrast, Reckles et al. reported that the incidence of scoliosis did not increase after surgery for CHD [2]. In either case, the prognosis of CHD patients has improved because of advances in cardiac care, and the frequency of surgery for scoliosis in this population increased as a result. When patients with a history of previous open-heart surgery also have decreased cardiac or pulmonary function, surgical treatment for scoliosis carries a relatively higher risk of inducing cardiopulmonary events.
We present the cases of two patients with scoliosis who had undergone previous surgery for CHD. We describe the procedures used to treat their scoliosis and the perioperative risks associated with these procedures.

\section{Materials and Methods}

Two patients who developed severe scoliosis after surgery for CHD were treated at our institution. We evaluated their spines according to three regions-proximal thoracic, main thoracic, and lumbar-with the apex of the proximal thoracic region between $\mathrm{T} 1$ and $\mathrm{T} 5$, the apex of the main thoracic region between $\mathrm{T} 5$ and $\mathrm{T} 12$, and the apex of the lumbar region between T12 and L5. We then evaluated cardiac status according to the three-group method described 
by Kadhim et al. [3], which is as follows: group S, patients with a functional single cardiac ventricle or whose cardiac anatomy necessitates single-ventricle palliative surgery; group $2 \mathrm{~N}$, patients with two ventricles who have no significant functional or anatomic cardiac abnormalities; and group $2 \mathrm{R}$, patients with two ventricles with significant residual cardiac abnormalities.

The present patients' preoperative anesthetic risk was determined by an anesthesiologist according to the criteria outlined by the American Society of Anesthesiologists (ASA). Cyanosis was defined as oxygen saturation $<94 \%$ at rest in room air. In both cases, pediatric cardiologists advised us to be aware of possible perioperative hemodynamic instability and, if present, to ensure continuous direct arterial blood pressure measurements and central venous catheterization for the administration of cardiovascular agonists, as needed, intraoperatively and postoperatively. Anesthesiologists performed this monitoring and minimized blood loss by using a cell saver intraoperatively to salvage any blood loss. In addition, pediatric cardiologists advised us to measure oxygen saturation regularly-not only intraoperatively and postoperatively but also preoperatively-to monitor for worsening heart function.

\section{Case Reports}

3.1. Case 1. A girl born with a right single ventricle had undergone bidirectional Glenn shunt and Fontan procedure during childhood. When she was 11 years old, she was diagnosed with scoliosis that gradually worsened until, at age 15, she was referred to our department. An anteroposterior radiograph showed an $83^{\circ}$ left convex main thoracic curve, a $40^{\circ}$ proximal thoracic curve, and a $68^{\circ}$ lumbar curve. The C7-central sacral vertical line (CSVL) was measured as the horizontal distance between the $\mathrm{C} 7$ plumb line and the CSVL, which was drawn through the center of the sacrum perpendicular to the iliac crests. The patient's C7-CSVL distance was within $20 \mathrm{~mm}$, indicating that coronal balance of the spine was maintained [4]. Thoracic kyphosis was $27^{\circ}$, and lumbar lordosis was $54^{\circ}$ (Figure 1). Side-bending tests showed $42 \%$ flexibility at the main thoracic curve. The patient suffered from restrictive lung function (forced vital capacity $48.6 \%$ and forced expiratory volume in $1 \mathrm{~s} 56.7 \%$ ), hypoxemia $\left(\mathrm{pO}_{2} 68.8 \mathrm{mmHg}\right.$ and $\mathrm{pCO}_{2} 35.8 \mathrm{mmHg}$ ), and chronic heart failure, which had resulted in a slightly elevated brain natriuretic peptide level $(38.4 \mathrm{pg} / \mathrm{dl})$ and aortic regurgitation. Her oxygen saturation was $<94 \%$ at rest in room air. Based on these findings, the patient's preoperative cardiac status was defined as group $S$, her anesthetic risk was classified as ASA 2, and she met the criterion for cyanosis. A reservoir of $1600 \mathrm{ml}$ of autologous blood was collected preoperatively.

Posterior release was performed with Ponte osteotomy at every level and dissection between the transverse process and the rib around the apex. Posterior fixation was performed with pedicle screws and $5.5 \mathrm{~mm}$ titanium composite metal rods. The rod-rotation technique was used for spinal correction. Operation time was $396 \mathrm{~min}$, and intraoperative blood loss was $1155 \mathrm{ml}$. The total amount of preoperatively collected autologous blood was returned to her during a period starting intraoperatively and ending postoperatively.

A postoperative radiograph showed $75 \%$ improvement in the patient's main thoracic curve. She remained in the intensive care unit for 2 days. On the first postoperative day, endotracheal intubation was maintained for respiration support, and the positive end-expiratory pressure was kept as low as possible to avoid deterioration of blood circulation caused by high pulmonary vascular resistance. On the second postoperative day, the patient's respiratory condition and blood pressure were stable, and she was extubated. There were no perioperative complications. At a 4-year follow-up visit, she was seen to have continued adequate curve correction and balance (Figure 2).

3.2. Case 2. A girl born with cyanosis and complete transposition of the great arteries had undergone an arterial switch operation, division of patent ductus arteriosus, and closure of patent foramen ovale soon after birth. At 3 years of age, she underwent surgery on her left coronary and pulmonary arteries. At age 5, she was diagnosed with scoliosis, which gradually worsened. The patient was referred to our department when she was 6 years old, and brace treatment was initiated. At 9 years of age, a left-sided abnormality of the abdominal skin reflex was noticed, and magnetic resonance imaging revealed an Arnold-Chiari malformation with syringomyelia, which was treated with suboccipital decompression.

At age 10, the patient's scoliosis had progressed further, resulting in a major thoracic curve $\left(95^{\circ}\right)$ with a central sacral vertical line $1.5 \mathrm{~cm}$ to the left of the vertical plumb line (Figure 3). Side-bending test results showed 22\% flexibility at the main thoracic curve. Prior to the latest operation, low cardiac output (left ventricular ejection fraction $45 \%-$ $50 \%)$ and hypotension $(85 / 53 \mathrm{mmHg}$ ) were present. Accordingly, the patient was considered to have group $2 \mathrm{R}$ preoperative cardiac status, and her anesthetic risk was classified as ASA 2. A reservoir of $2340 \mathrm{ml}$ of autologous blood was collected preoperatively.

The same operative technique as was used in case 1 was performed for this patient but with different instruments. The operative time was $388 \mathrm{~min}$, and intraoperative blood loss was $1806 \mathrm{ml}$. The entire amount of preoperatively collected autologous blood was returned to her starting intraoperatively and ending postoperatively. Postoperative radiography showed $74 \%$ improvement in the patient's main thoracic curve, and the thoracic kyphosis was corrected from $60^{\circ}$ to $31^{\circ}$.

The patient remained in the intensive care unit for 3 days. On the first postoperative day, her systolic blood pressure fell temporarily to 50-60 mmHg. After semi-Fowler's positioning and a rapid intravenous drip infusion, her systolic blood pressure was maintained at around $100 \mathrm{mmHg}$. On the second day, the patient's blood pressure was stable. Her respiratory rate, tidal volume, and oxygenation were maintained unless her ventilation was switched from synchronized intermittent mandatory ventilation to spontaneous respiration, so she was extubated. After extubation, because the patient's oxygenation was somewhat poor, oxygen administration 

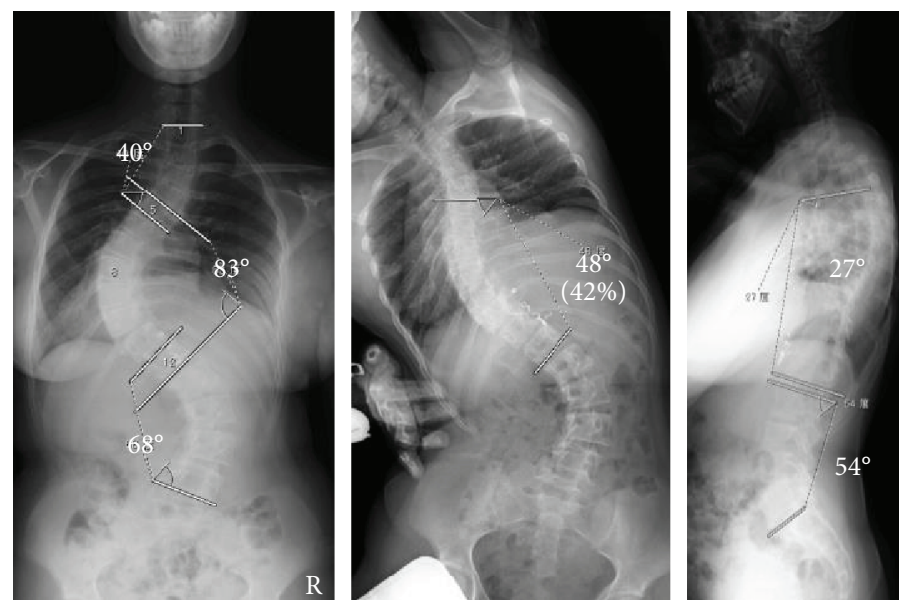

Left bending

FIGURE 1: Case 1: preoperative radiographs (from left to right: anteroposterior, side-bending, lateral). Anteroposterior radiograph shows an $83^{\circ}$ left convex main thoracic curve, a $40^{\circ}$ proximal thoracic curve, and a $68^{\circ}$ lumbar curve. Side-bending radiograph shows $42 \%$ flexibility at the main thoracic curve. Lateral radiograph shows thoracic kyphosis of $27^{\circ}$ and lumbar lordosis of $54^{\circ}$.
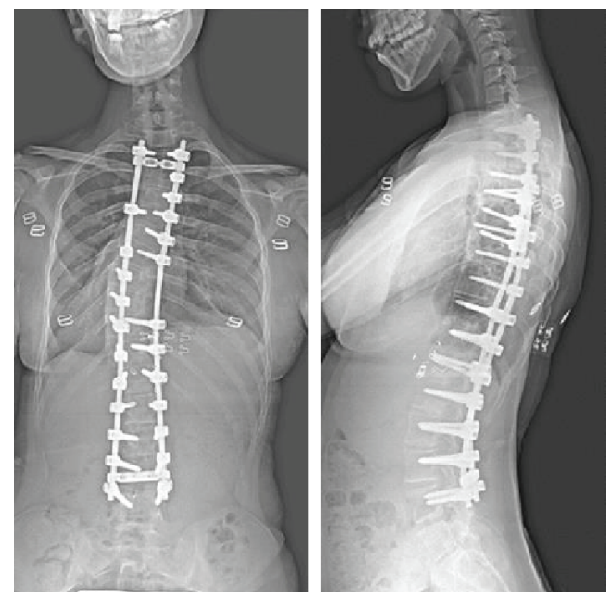

FIGURE 2: Case 1: postoperative radiographs (anteroposterior, lateral) 4 years after surgery show adequate curve correction and balance.

was continued until the third postoperative day. Thereafter, the patient experienced no perioperative complications. At the 2-year follow-up visit, she exhibited adequate curve correction and balance (Figure 4).

\section{Discussion}

Several studies have discussed the incidence of scoliosis after open-heart surgery $[5,6]$. Kawakami et al. [5] reported that the incidence of scoliosis is higher among patients with CHD than in the general population $(2 \%-19 \%$ vs. $2 \%-3 \%$, respectively). These authors also reported that markedly fewer patients exhibited scoliosis before cardiac surgery than afterward (11 vs. 63 patients, respectively). Ruiz-Iban et al. [6] reported that many patients with $\mathrm{CHD}$, especially those at age $<18$ months, develop scoliosis after undergoing procedures that included median sternotomy. These findings suggest that not only the Arnold-Chiari malformation but also the cardiac operation may have contributed to the development of scoliosis in the patient described in our case 2. In contrast, other studies have found that cardiac surgery does not increase the incidence of scoliosis [2, 7]. Further study is needed to confirm the risk factors for scoliosis.

Correcting severe scoliosis involves long surgical procedures and is associated with significant blood loss. Hence, intraoperative and postoperative complications are common, especially in patients with a history of CHD surgery. Hedequist et al. reported that patients with Fontan circulation (with poor systemic circulation and poor cardiac status) experienced a variety of complications after surgery for scoliosis, including paraparesis caused by vascular infarction affecting the midthoracic spinal cord and acute tubular necrosis [8]. Another study found that, during posterior spinal fusion for idiopathic scoliosis in patients with Fontan physiology, the venous pressure was markedly elevated because of being in the prone position, resulting in a prolonged prothrombin time and increased blood loss [9]. Evans et al. also suggested that patients with Fontan circulation have high venous pressure, particularly while in the prone position. Hence, operations requiring the prone position in these patients may result in significant blood loss [10]. Considering these concerns, we took extra care during the operation to avoid excessive blood loss in case 1.

In contrast, several researchers have reported a lower intraoperative cardiac index in patients undergoing surgery in the prone position $[11,12]$. Brown et al. described changes in the cardiac indices of children undergoing scoliosis surgery in the prone position [13]. The authors used a Jackson frame in all cases, which reduced the risk of elevated venous pressure, but the patients' cardiac indices still fell. Therefore, the authors thought that increased intrathoracic pressure may be related to the lower cardiac index. Our patient in case 2 had low cardiac output, and therefore it was important to conduct an adequate preoperative cardiac evaluation. 

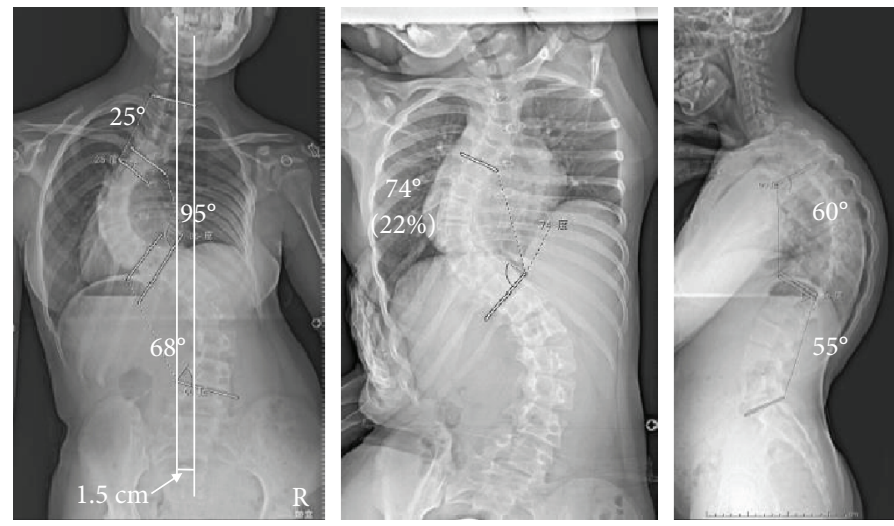

Left bending

FIGURE 3: Case 2: preoperative radiographs (from left to right: anteroposterior, side-bending, lateral). Anteroposterior radiograph shows a major thoracic curve of $95^{\circ}$ with a central sacral vertical line $1.5 \mathrm{~cm}$ to the left of the vertical plumb line. Side-bending radiograph shows $22 \%$ flexibility at the main thoracic curve. Lateral radiograph shows thoracic kyphosis of $60^{\circ}$ and lumbar lordosis of $55^{\circ}$.
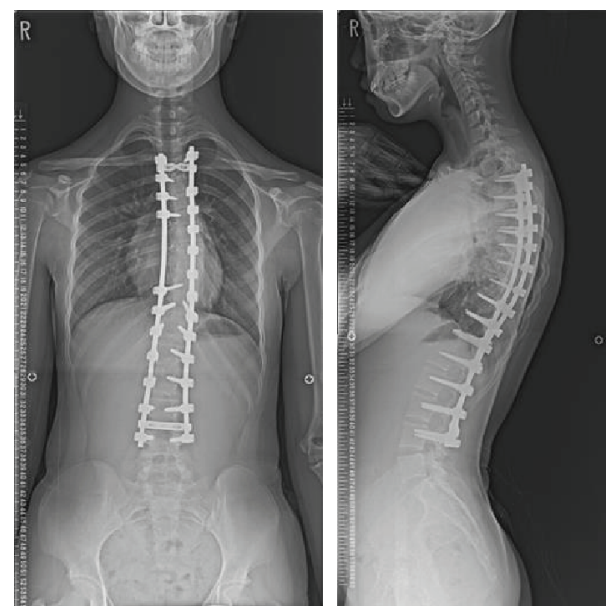

FIGURE 4: Case 2: radiographs (anteroposterior, lateral) 2 years after surgery show adequate curve correction and balance.

Because surgery for scoliosis affects cardiopulmonary function, accurate preoperative assessment and appropriate intraoperative care are especially important for $\mathrm{CHD}$ patients undergoing scoliosis surgery. Perioperative care is also important for these patients. Particularly, excessive blood loss tends to occur in patients with Fontan circulation. Hypotensive anesthesia is often useful to prevent bleeding, although CHD patients with unstable hemodynamics can easily develop hypoperfusion. Evans et al. reported that operations during which the patient must remain prone for more than $4 \mathrm{~h}$ were associated with an increased risk of bleeding and hypotension, even when the patient was initially hemodynamically stable [10]. Patients with Fontan circulation (as in case 1) receive lifelong anticoagulant therapy, which makes the perioperative use of hemostatic agents difficult. Both operations described in this study involved long operation times. Because there was close collaboration between the pediatric cardiologist and anesthetist, adequate preoperative evaluation, and appropriate perioperative care, the patients had satisfactory outcomes.
The main limitation of our study is that it involved only two cases. Further studies are needed to determine definitively whether our approach reduces the risk of perioperative complications in CHD patients undergoing surgery for scoliosis.

\section{Conclusion}

The prognosis of CHD patients has improved, and many patients can now undergo surgery safely for their scoliosis. We performed scoliosis surgery in two patients with $\mathrm{CHD}$ and obtained satisfactory results, with no serious complications in either patient. Nevertheless, we remain vigilant and cautious as CHD patients sometimes exhibit unexpected hemodynamics and respiratory conditions. We believe that the combination of appropriate surgical technique and cooperative teamwork among experts is the key to success in such cases.

\section{Ethical Approval}

All procedures performed in studies involving human participants were in accordance with the ethical standards of the Akita University Research Committee and with the 1964 Helsinki Declaration and its later amendments or comparable ethical standards.

\section{Consent}

Informed consent was obtained from both individual participants included in the study.

\section{Conflicts of Interest}

We have no conflicts of interest.

\section{Acknowledgments}

We thank Rebecca Tollefson, DVM, from Edanz Group (https://www.edanzediting.com/ac) for editing a draft of this manuscript. 


\section{References}

[1] F. C. Van Biezen, P. A. Bakx, V. H. De Villeneuve, and W. C. Hop, "Scoliosis in children after thoracotomy for aortic coarctation," The Journal of Bone \& Joint Surgery, vol. 75, no. 4, pp. 514-518, 1993.

[2] L. N. Reckles, H. A. Peterson, W. H. Weidman, and A. J. Bianco Jr., "The association of scoliosis and congenital heart defects," The Journal of Bone \& Joint Surgery, vol. 57, no. 4, pp. 449-455, 1975.

[3] M. Kadhim, E. Spurrier, D. Thacker, C. Pizarro, and W. G. Mackenzie, "Scoliosis surgery in children with congenital heart disease," Spine, vol. 39, no. 3, pp. E211-E218, 2014.

[4] B. S. Richards, A. Scaduto, K. Vanderhave, and R. Browne, "Assessment of trunk balance in thoracic scoliosis," Spine, vol. 30, no. 14, pp. 1621-1626, 2005.

[5] N. Kawakami, K. Mimatsu, M. Deguchi, F. Kato, and S. Maki, "Scoliosis and congenital heart disease," Spine, vol. 20, no. 11, pp. 1252-1255, 1995.

[6] M. A. Ruiz-Iban, J. Burgos, H. J. Aguado et al., "Scoliosis after median sternotomy in children with congenital heart disease," Spine, vol. 30, no. 8, pp. E214-E218, 2005.

[7] H. H. Feiz, A. Afrasiabi, R. Parvizi, A. Safarpour, and R. F. Fouladi, "Scoliosis after thoracotomy/sternotomy in children with congenital heart disease," Indian Journal of Orthopaedics, vol. 46, no. 1, pp. 77-80, 2012.

[8] D. J. Hedequist, J. B. Emans, and J. E. Hall, "Operative treatment of scoliosis in patients with a Fontan circulation," Spine, vol. 31, no. 2, pp. 202-205, 2006.

[9] M. B. Rafique, E. A. E. Stuth, and J. C. Tassone, "Increased blood loss during posterior spinal fusion for idiopathic scoliosis in an adolescent with Fontan physiology," Paediatric Anaesthesia, vol. 16, no. 2, pp. 206-212, 2006.

[10] S. Evans, A. Ramasamy, D. S. Marks et al., "The surgical management of spinal deformity in children with a Fontan circulation," The Bone \& Joint Journal, vol. 96-B, no. 1, pp. 94-99, 2014.

[11] M. Yokoyama, W. Ueda, M. Hirakawa, and H. Yamamoto, "Hemodynamic effect of the prone position during anesthesia," Acta Anaesthesiologica Scandinavica, vol. 35, no. 8, pp. 741-744, 1991.

[12] P. S. Sudheer, S. W. Logan, B. Ateleanu, and J. E. Hall, "Haemodynamic effects of the prone position: a comparison of propofol total intravenous and inhalation anaesthesia," Anaesthesia, vol. 61, no. 2, pp. 138-141, 2006.

[13] Z. E. Brown, M. Görges, E. Cooke, S. Malherbe, G. A. Dumont, and J. M. Ansermino, "Changes in cardiac index and blood pressure on positioning children prone for scoliosis surgery," Anaesthesia, vol. 68, no. 7, pp. 742-746, 2013. 


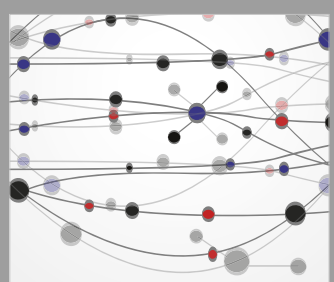

The Scientific World Journal
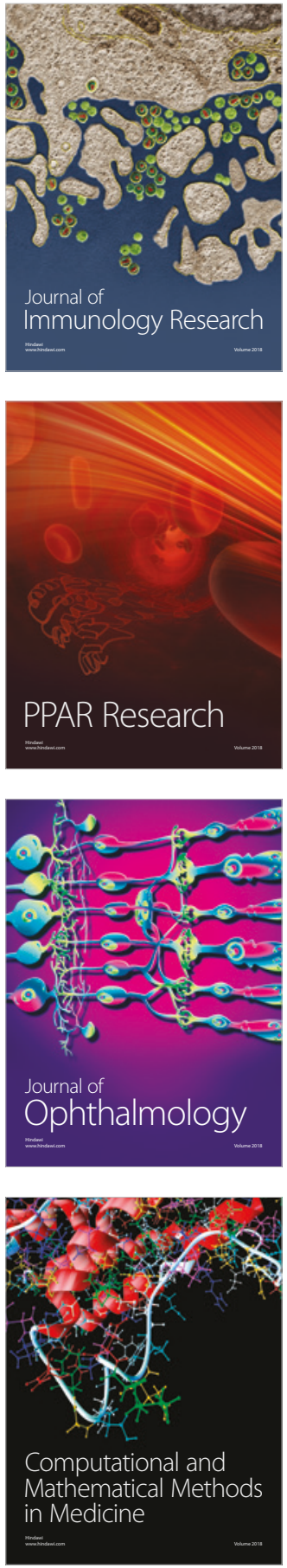

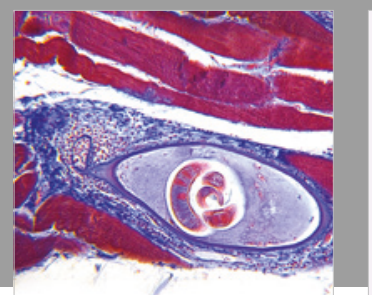

Gastroenterology Research and Practice

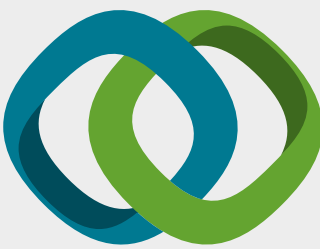

\section{Hindawi}

Submit your manuscripts at

www.hindawi.com
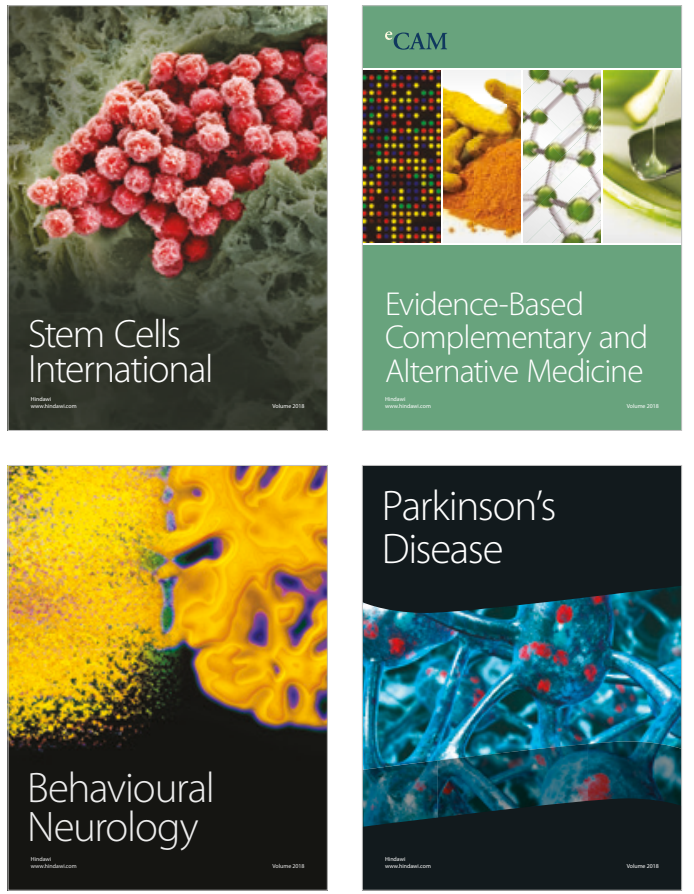

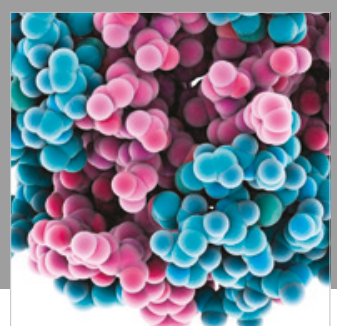

ournal of

Diabetes Research

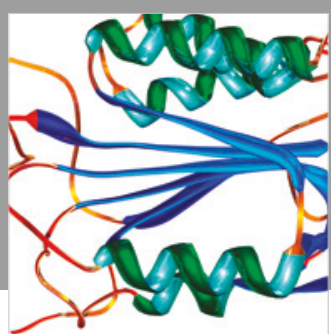

Disease Markers
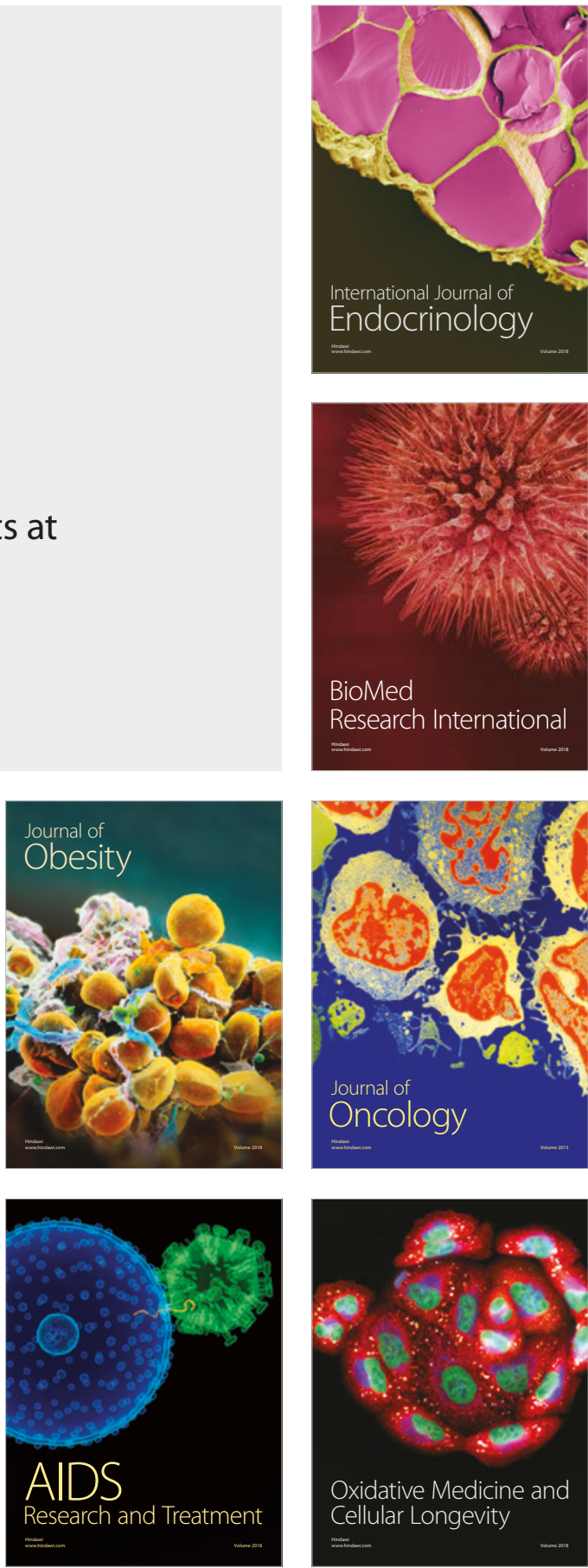